\title{
openheart Catheter strategy to ease the procedure and reduce radiation exposure when requiring neck access
}

\author{
Rouven Kubicki (D) , ${ }^{1}$ Johanna Hummel, ${ }^{1}$ René Höhn, ${ }^{1}$ Kevin Müller, ${ }^{2}$ \\ Brigitte Stiller, ${ }^{1}$ Jochen Grohmann ${ }^{1}$
}

\begin{abstract}
- Additional material is published online only. To view please visit the journal online (http://dx.doi.org/10.1136/ openhrt-2020-001267).
\end{abstract}

To cite: Kubicki R, Hummel J, Höhn R, et al. Catheter strategy to ease the procedure and reduce radiation exposure when requiring neck access. Open Heart 2020;7:e001267. doi:10.1136/ openhrt-2020-001267

Received 14 February 2020 Revised 30 March 2020 Accepted 11 May 2020

Check for updates

(c) Author(s) (or their employer(s)) 2020. Re-use permitted under CC BY-NC. No commercial re-use. See rights and permissions. Published by BMJ.

${ }^{1}$ Department of Congenital Heart Disease and Paediatric Cardiology, University Heart Center Freiburg - Bad Krozingen, Medical Centre University of Freiburg, Faculty of Medicine, University of Freiburg, Freiburg, Germany, Freiburg, Germany

${ }^{2}$ Department of Occupational Safety, Medical Centre University of Freiburg, Faculty of Medicine, University of Freiburg, Freiburg, Germany, Freiburg, Germany

Correspondence to Dr Rouven Kubicki; Rouven. kubicki@universitaetsherzzentrum.de

\begin{abstract}
Objectives To assess the potential occupational radiation reduction and technical feasibility in patients rotated $180^{\circ}$ (upside-down) when requiring neck access for transcervical or trans-subclavian catheterisation.

Methods Upside-down positioning is defined as rotating patients in supine position by $180^{\circ}$, so that the feet come to rest where the head would otherwise be. We retrospectively evaluated all these procedures performed between March 2016 and May 2019. Furthermore, two different phantoms (paediatric and adult) were used prospectively to quantify the occupational dose between conventional or upside-down positioning. In this context, ambient dose equivalents were measured using real-time dosimeters. Three different projection angles were applied. Results 44 patients with median age and body weight of 1.0 year (range $0-56$ ) and $9.5 \mathrm{~kg}$ (range 1.3-74.3) underwent 63 procedures positioned upside-down. This position proved advantageous for practical reasons, since the length of the examination table could be optimally used. Additionally, it resulted in a significantly lower overall ambient dose equivalent for the primary operator (PO) of $94.8 \%$ (mean: $2569 \pm 807$ vs $135 \pm 23 n S v ; p<0.01$ ) in the adult, and of $65.5 \%$ (mean: $351 \pm 104$ vs $121 \pm 56 \mathrm{nSv}$; $\mathrm{p}<0.01$ ) in the paediatric phantom, respectively. Conclusion Upside-down positioning facilitates handling in a straightforward manner when access from the neck is required. Moreover, it significantly reduces local radiation exposure for the $\mathrm{PO}$ in the paediatric and, most impressively, in the adult phantom.
\end{abstract}

\section{INTRODUCTION}

Due to major advances in diagnostics, perioperative care and surgical techniques, at present $70 \%-95 \%$ of infants with congenital heart disease survive into adulthood. Nevertheless, there is still a high rate of long-term morbidity that often requires catheter interventions. Considerable progress has been made in this field, leading to a shift from primary diagnostics to numerous or repeated treatment modalities. ${ }^{12}$

Access via the groin is most frequent in congenital cardiac catheterisation. However, alternative vascular approaches like the jugular vein can be advantageous or even

\section{Key questions}

What is already known about this subject?

- Vascular access from the neck as an alternate route is mandatory for certain transcatheter interventions to alleviate congenital and structural heart disease. However, the positioning of the team members involved may not be well-suited for performing procedures from the neck, which can entail greater occupational radiation exposure.

What does this study add?

- Positioning the patient lying on his back rotated by $180^{\circ}$ (upside-down) on the catheter table facilitates interventions via neck access and proved to be practical, enabling optimal use of the catheter table with improved sterile working conditions. Additionally, the primary operator's local radiation exposure was reduced significantly in the paediatric, and most impressively in the adult phantom as demonstrated by bench-test simulation.

How might this impact on clinical practice?

- In modern catheterisation laboratories for congenital heart disease, this new patient position might become routine in cases requiring neck access, since it reduced the operators' radiation exposure and enabled more comfortable sterile working conditions.

mandatory in children and adults presenting stenotic groin vessels or certain anatomy like single-ventricle physiology or vena azygos drainage. ${ }^{3}$ Access via the jugular vein is also considered sensible for percutaneous pulmonary valve implantation, especially in young children. ${ }^{45}$ The carotid artery enables easy and alternative access, especially in small newborns with pulmonary atresia requiring ductal stenting. ${ }^{6}$

From the technical point of view, neck access is problematic concerning the handling of long sheaths, exchange wires and delivery systems. Furthermore, it is more demanding to ensure cleanliness and sterile working conditions. Our solution was to rotate the patient in supine position by $180^{\circ}$ 
on the examination table (change head and feet to an upside-down position) to create more comfortable sterile working conditions.

This report describes our preliminary clinical experience with this positional change in terms of feasibility and handling for various treatment modalities. In the second step, we explored the new upside-down position regarding the radiation exposure for staff, using different phantoms (paediatric and adult) to quantify the occupational dose between the two different patient positions for bench-test simulation.

\section{METHODS}

\section{Study population}

We retrospectively evaluated all consecutive paediatric and adult patients who underwent cardiac catheterisation under conscious sedation or general anaesthesia via neck access with upside-down positioning between March 2016 and May 2019. Of note, upside-down positioning results in a changed angiographic orientation: cranial becomes caudal in both the A and B planes; in addition, right is right and left is left in the A plane (break with the standard X-ray convention). On the Siemens Artis zee, fluoroscopy can be digitally inverted and mirrored live during image acquisition, if the examiner requests so. Upside-down position is shown in online supplementary 1 . Depending on the indication, transcervical access via the common carotid artery and internal jugular vein as well as trans-subclavian access had been established percutaneously. Baseline demographics comprised age, gender, body surface area and patients' diagnoses. Moreover, the indication for catheterisation and procedurerelated data were reviewed, including the individual's X-ray exposure as fluoroscopy times and dose area products.

\section{Measurements of occupational dose}

All series were measured in the same catheterisation laboratory using an Artis Zee biplane angiography unit (Siemens Healthcare GmbH, Forchheim, Germany), equipped with two $30 \times 40 \mathrm{~cm}$ flat panel detectors. Acquisitions took place in biplane with fixed radiation exposures lasting $4 \mathrm{~s}$. The distance between the detector and phantom was kept as close as possible during the acquisitions based on the ALARA ('as low as reasonably achievable') principle. Two different recording programmes were used for child and adult set-ups: the paediatric setting was selected for acquisition at 7.5 frames/s, with $73 \mathrm{kV}$ tube voltage and $410 \mathrm{~mA}$ tube under automatic dose rate control and $0.2 \mathrm{~mm} \mathrm{Cu}$ filtration. The adult setting was selected for acquisition at 7.5 frames/s, with $70 \mathrm{kV}$ tube voltage and $800 \mathrm{~mA}$ tube under automatic dose rate control and $0.3 \mathrm{~mm} \mathrm{Cu}$ filtration. Automatic exposure control automatically adjusts the following parameters depending on each phantom and the C-arm angulation: tube current, focal spot, filtration, exposure time and tube voltage. We used the combined applications to reduce exposure analytics as dose monitoring technology.

Two different anthropomorphic Rando Alderson phantoms (Radiology Support Devices, California, USA) and one spherical body were used to simulate X-ray attenuation and scatter under a direct beam. One phantom (Braden) represented a 3-7-year-old child with height and body weight of $119 \mathrm{~cm}$ and $19 \mathrm{~kg}$. Another phantom (Lawrence) corresponded to an adult. The phantoms were placed in supine position on the catheter table. Both phantoms were limbless, with their torsos consisting of natural human bones embedded in soft tissue-equivalent material. The phantom's absorption and scattering were equivalent to those in humans. The spherical body is a phantom normally used for expert appraisals in the context of radiation-protection ordinance as test specimen for the acceptance test on an X-ray and X-ray fluoroscopic system.

In the conventional set-up, the phantom is placed in supine position; head and feet come to rest in the designated location. Upside-down positioning means maintaining the supine position, but rotating the phantom by $180^{\circ}$ so that the feet come to rest where the head would be during conventional cardiac catheterisation. Instead of the primary operator (PO) being positioned between the two C-arms of the X-ray unit, in the new set-up, the $\mathrm{PO}$ is on the right side standing along the long side of the examination table. Standard radiation protection measures consisting of a leaded glass pane and tablesuspended drapes were used recreating real-life conditions as closely as possible. We measured ambient dose equivalents using real-time dosimeters (Thermo Scientific FH 40 G-L10, Germany). To obtain good measurement results, the radiation must strike the square marking on the front of the FH $40 \mathrm{G}-\mathrm{L} 10$ up to an angle of $75^{\circ}$ to the longitudinal axis of the device. The measuring range of the device is from $10 \mathrm{nSv} / \mathrm{h}$ to $100 \mathrm{mSv} / \mathrm{h}$ (30 keV to 4.4 $\mathrm{MeV}$ ). The sensitivity range of the device is from $100 \mathrm{nSv}$ to $1 \mathrm{~Sv}$. The dosimeter was attached at the front of the test persons' lead-protective aprons at a height of $1.40 \mathrm{~m}$.

The staff was represented by authors-as genuine subjects placed at four previously defined measuring points inside the cardiac catheterisation laboratory in each setting. Based on radiation-protection best practice, we wore a lead apron, thyroid shields, lead caps and eyewear. An additional portable protection shield protected the anaesthetist. Radiation exposure was determined for the PO, first assistant, anaesthetist and one nurse. Operators' standing position in relation to the X-ray source and patient phantom mimicks a typical catheterisation scenario via neck access. The precise arrangement of our experimental set-up is shown in figure 1 .

Using the biplane mode, three different standard projection angles were applied for the A plane with the corresponding $\mathrm{B}$ plane remaining in the lateral projection (left anterior oblique (LAO) 90 ${ }^{\circ}$ ): first, frontal (posterior-anterior) and lateral view; second, 

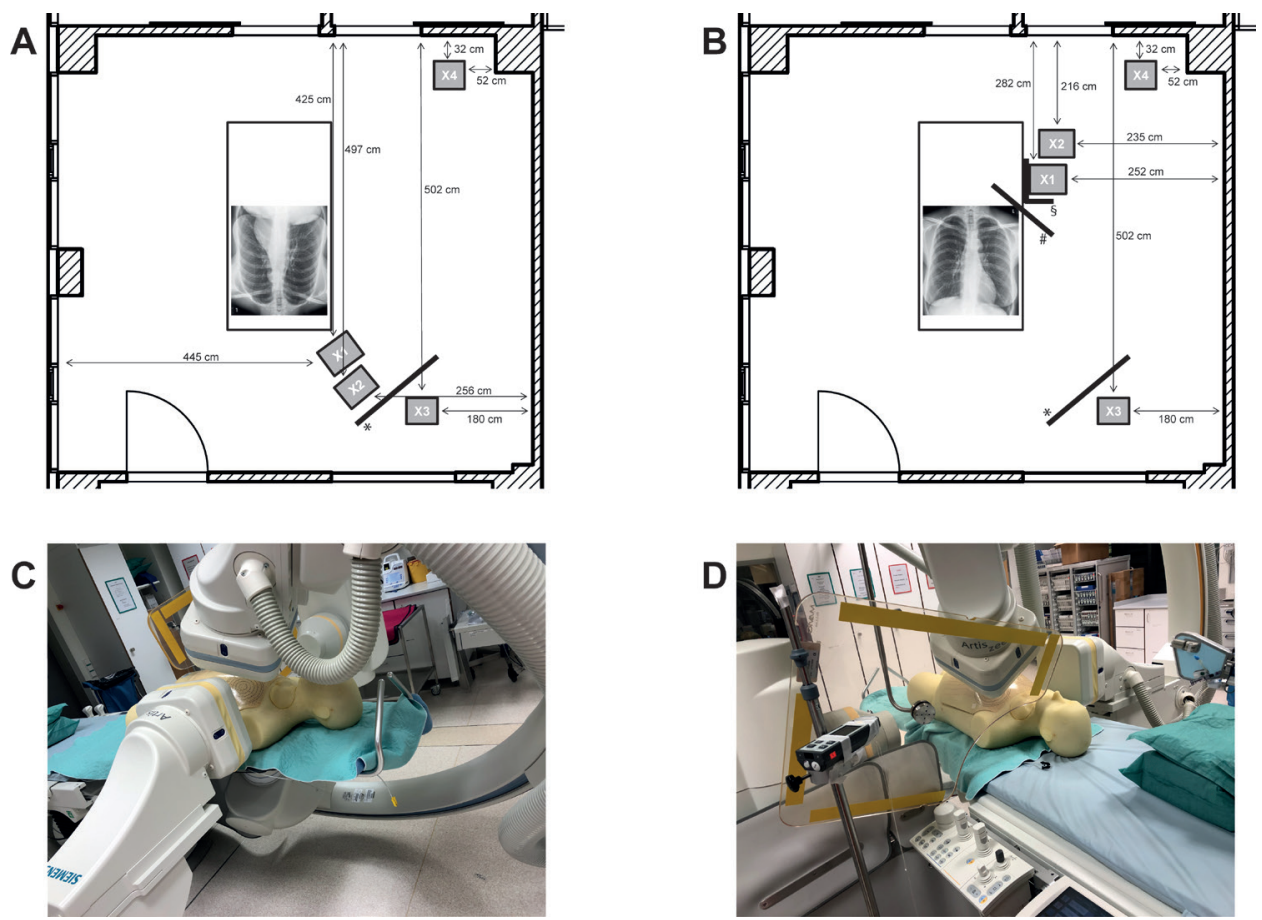

Figure 1 (A) Conventional set-up: the phantom is placed in the supine position, head and feet come to rest in the designated location. Four defined measuring points: $X 1=P O, X 2=$ first assistant, $X 3=$ intensivist/anaesthetist and $X 4=$ nurse. ${ }^{*}$ Portable protection shield. (B) Upside-down positioning, instead of the PO being left between the two C-arms of the unit, in the new set-up the PO is on the long side of the examination table as usual. *Portable protection shield, \#leaded glass pane and §tablesuspended drapes. (C) Conventional cranial-LAO/lateral projection. (D) upside-down positioning cranial-LAO/lateral projection. LAO, left anterior oblique; PO, primary operator.

cranial-LAO $30^{\circ} / 30^{\circ}$ and lateral view; third, cranial right anterior oblique (cranial-RAO) $30^{\circ} / 20^{\circ}$ and lateral view. In each angulation, we took three measurements each and from these we formed the mean value for both the conventional and upside-down positioning of each patient-phantom.

\section{Statistical analysis}

Data collection took place over 3 days. The values measured on each day and each phantom with the corresponding angulations were averaged separately over three measurements and are presented as mean values with their SD. Student's t-test was used to assess for differences. Patient data were analysed descriptively. In this study, the relative change in radiation exposure was determined by recording each staff member's effective radiation dose. Continuous data were summarised by median, quartiles $(\mathrm{IQR})$ and range or as mean $\pm \mathrm{SD}$. Categorical variables are presented as absolute numbers and percentages. All data were analysed using SPSS V.24.0 (SPSS, Washington, DC).

\section{RESULTS}

\section{Clinical application}

We carried out this upside-down positioning in our centre successfully during 63 procedures in 44 patients with an age and body weight of median 1.0 year (range $0-56$ ) and $9.5 \mathrm{~kg}$ (range 1.3-74.3). Some patients require more than one procedure, especially those with single-ventricle physiology. Patient characteristics and procedure-related data are shown in tables 1 and 2. In single-ventricle lesions, the main procedures were pulmonary artery

Table 1 Patient $(n=44)$ characteristics and procedurerelated data $(n=63)$

\begin{tabular}{|c|c|}
\hline Age, median (IQR), years & $1.0(0.0-3.8,0.0-56)$ \\
\hline Sex, female/male, $\%$ & $52.3 / 47.7$ \\
\hline Weight, median (IQR), kg & $9.5(4.1-17.0,1.3-74.3)$ \\
\hline Height, median (IQR), cm & $76.0(50-111,40-80)$ \\
\hline Body surface area, median (IQR), $\mathrm{m}^{2}$ & $0.45(0.24-0.72,0.12-1.93)$ \\
\hline \multicolumn{2}{|l|}{ Access, n (\%) } \\
\hline Right jugular vein & $34(53.9)$ \\
\hline Left jugular vein & $17(27.0)$ \\
\hline Right subclavian vein & $2(3.2)$ \\
\hline Left subclavian vein & $1(1.6)$ \\
\hline Right common carotid artery & $7(11.1)$ \\
\hline Left common carotid artery & $2(3.2)$ \\
\hline Sheath size, median (IQR), Fr & $4.0(4.0-5.7,2.7-18.0)$ \\
\hline Procedure time (mean $\pm \mathrm{SD}$ ), min & $217 \pm 12$ \\
\hline Fluoroscopy time (mean $\pm \mathrm{SD}$ ), min & $28.1 \pm 3.1$ \\
\hline $\begin{array}{l}\text { Dose area product (mean } \pm S D \text { ), } \\
\mu G{ }^{\star}{ }^{*} \mathrm{C} 2\end{array}$ & $1845 \pm 705$ \\
\hline General anaesthesia, yes/no, \% & $59 / 41$ \\
\hline
\end{tabular}




\begin{tabular}{|c|c|c|}
\hline Physiology & Diagnosis & Procedures \\
\hline $\begin{array}{l}\text { SV s/p stage I } \\
(2.3 \%)\end{array}$ & HLHS/HLHC $(n=1)$ & Branch PA stenting $(\mathrm{n}=1)$. \\
\hline $\begin{array}{l}\text { SV s/p stage II } \\
(34.1 \%)\end{array}$ & $\begin{array}{l}\text { HLHS / HLHC }(n=9) \\
\text { AVSD dysbalance }(n=3) \\
\text { DILV }(n=3)\end{array}$ & $\begin{aligned} & \text { Branch PA intervention } \\
& (\mathrm{n}=18) . \\
& \text { SVC stenting }(\mathrm{n}=1) . \\
& \text { Venous collateral closure } \\
& (\mathrm{n}=7) .\end{aligned}$ \\
\hline \multirow[t]{6}{*}{$\begin{array}{l}\text { BV } \\
(63.6 \%)\end{array}$} & $\begin{array}{l}\text { IVC anomaly, or } \\
\text { inappropriate vascular } \\
\text { calibre of femoral/iliacal } \\
\text { vessels }(n=12)\end{array}$ & $\begin{array}{l}\text { PPVI }(n=7) \text {. } \\
\text { ASD device closure } \\
(n=3) \text {. } \\
\text { IVC stenting }(n=1) \text {. } \\
\text { Branch PA stenting }(n=2) \text {. } \\
\text { BAP pulmonary vein } \\
\text { stenosis }(n=2) \text {. }\end{array}$ \\
\hline & SVC syndrome $(n=3)$ & SVC stenting $(\mathrm{n}=3)$ \\
\hline & Pulmonary atresia $(n=2)$ & - PDA stenting $(\mathrm{n}=4)$. \\
\hline & $\begin{array}{l}\text { Aortic arch hypoplasia } \\
(n=2)\end{array}$ & $\begin{array}{l}\text { Aortic arch stenting } \\
(\mathrm{n}=2) .\end{array}$ \\
\hline & $\begin{array}{l}\text { TAPVD with obstruction } \\
(n=3)\end{array}$ & $\begin{array}{l}\text { Vertical vein stenting } \\
(n=2) \text {. } \\
\text { Venous duct stenting } \\
(n=2) .\end{array}$ \\
\hline & $\begin{array}{l}\text { PAPVD }(n=1) \\
\text { SCT }(n=2) \\
\operatorname{mVSD}(n=1) \\
\text { ECM0 }(n=1) \\
\text { Pulmonary vein stenosis } \\
(n=1)\end{array}$ & $\begin{aligned} & \text { Vertical vein closure } \\
&(n=1) . \\
& \text { Coiling of feeding arteries } \\
&(n=2) . \\
& \text { VSD closure }(n=1) . \\
& \text { Placement cannula }(n=1) . \\
& \text { Diagnostic }(n=1) / B A P \\
& \text { pulmonary vein stenosis } \\
&(n=2) .\end{aligned}$ \\
\hline
\end{tabular}

ASD, atrial septal defect; AVSD, atrioventricular septal defect; BAP, balloon angioplasty; BV, biventricular heart; DILV, double inlet left ventricle; ECMO, extracorporeal membrane oxygenation; HLHS/HLHC, hypoplastic left heart syndrome/complex; IVC, inferior vena cava; mVSD, muscular ventricular septal defect; PA, pulmonary artery; PAPVD, partial anomalous pulmonary venous drainage; PDA, patent ductus arteriosus; PPVI, percutaneous pulmonary valve implantation; SCT, sacrococcygeal teratoma; SV, single ventricle; SVC, superior vena cava; TAPVD, total anomalous pulmonary venous drainage; VSD, ventricular septal defect.

interventions $(n=19)$ and the closure of venous collaterals $(n=7)$. The majority of patients with biventricular physiology had inferior vena cava (IVC) anomalies $(n=12)$ or superior vena cava (SVC) syndrome $(n=3)$ as the reason for upside-down positioning, including some neonatal interventions where a transcervical route was preferable (eg, stenting the arterial duct or aortic arch; figure 2). The most frequent access points were the right jugular vein $(53.9 \%)$, left jugular vein $(27.0 \%)$ and right common carotid artery $(11.1 \%)$. No access-related complications occurred. Sheath sizes ranged from 2.7 to $18 \mathrm{Fr}$. Mean fluoroscopy times and dose-area products were $28.1 \pm 3.1 \mathrm{~min}$ and $1845 \pm 705 \mathrm{cGy}^{*} \mathrm{~cm}^{2}$. Upsidedown positioning enabled more efficient use of structural radiation protection (eg, ceiling-mounted shields), while at the same time the PO was positioned in a low-scattered area. The length of the examination table was also more effectively used simplifying the handling of long sheaths, exchange wires and delivery systems and ensuring sterile working conditions. The perspective change in imaging (break with the standard X-ray convention) took some getting used to, but was implemented well. We experienced no projection-related complications.

\section{Experimental set-up}

Upside-down patient positioning resulted in a significant reduction in ambient dose equivalent for the PO of $94.8 \%$ (mean: $2569 \pm 807$ vs $135 \pm 23 \mathrm{nSv}, \mathrm{p}<0.01$ ) in the adult phantom, $65.5 \%$ (mean: $351 \pm 104$ vs $121 \pm 56 \mathrm{nSv}$, $\mathrm{p}<0.01$ ) in the paediatric phantom and $97.8 \%$ (mean: $3907 \pm 883$ vs $126 \pm 13 \mathrm{nSv}, \mathrm{p}<0.01$ ) in the spherical model, respectively. Table 3 summarises the radiation exposure comparing the three different models averaged over all three angulations. In both the adult and spherical model, radiation exposure to the $\mathrm{PO}$ was about 10-fold higher than that of the paediatric model. We documented similarly low ambient dose equivalent values $(\leq 200 \mathrm{nSv})$ in all three models after an upside-down positioning. Of note, the first assistant's exposure to radiation increased significantly in all three models after switching to upside-down positioning. However, the measured doses were under $300 \mathrm{nSV}$, and thus lower than the benefit for the PO. No matter how we had positioned the patient phantoms, neither nurse nor anaesthetists were exposed to relevant radiation.

Table 4 shows the comparison of ambient dose equivalent values at different tube settings. Positioned conventionally, cranial-LAO projections (adult: 3540 \pm 196 ; paediatrics: $468 \pm 20 \mathrm{nSv}$; spherical: $4781 \pm 194)$ are most radiation-intensive for the $\mathrm{PO}$ in all models. When switching to upside-down, cranial-LAO generated the lowest PO's ambient dose equivalent in the adult phantom (106 $\pm 6 \mathrm{nSv})$ and cranial-RAO $(57 \pm 14 \mathrm{nSv})$ in the paediatric phantom. No particular position offered additional radiation protection for the first assistant (see also figure 3 ).

\section{DISCUSSION}

Alternative vascular approaches are advantageous or even mandatory in patients presenting certain anatomy. ${ }^{6-9}$ In this series, $34.1 \%$ of patients had stage II Glenn palliation in single-ventricle physiology requiring access from the neck if the SVC and pulmonary arteries were targeted. In addition, access to the jugular and subclavian veins is an option if the IVC is absent, aberrant, stenotic, thrombosed or simply too small, which may also affect the femoral and pelvic vessels. Moreover, access from the neck may enable a more practical, direct route to specific anatomical structures and difficult-to-reach lesions. In this context, the carotid approach should also be highlighted, as it makes a direct route to the aorta possible. In young infants, the 

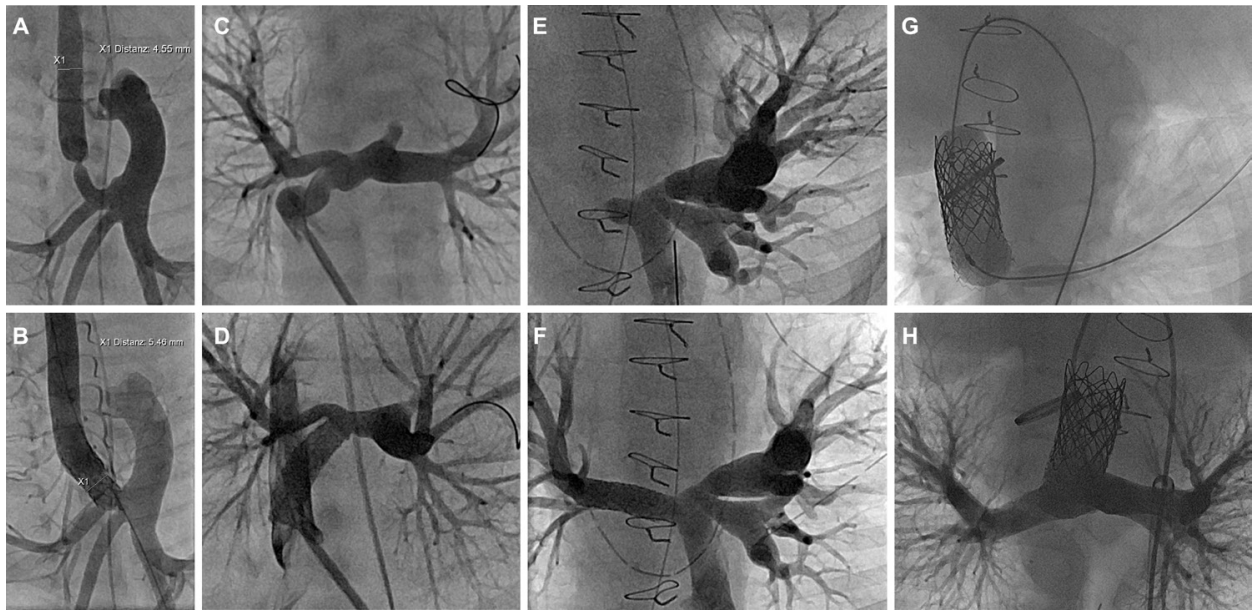

Figure 2 Transcervical catheterisation using upside-down positioning. (A/B) Preterm infant weighing $1.6 \mathrm{~kg}$ with distal aortic arch hypoplasia and severe coarctation stented with a redilatable peripheral stent via the right common carotid artery. (C/D) Angiography via the right common carotid artery demonstrates a tortuous vertical duct in a $3.6 \mathrm{~kg}$ neonate with congenitally corrected transposition of the great arteries, and the result following duct stenting. (E/F) Angiography via the right jugular vein reveals a completely occluded left pulmonary artery following stage II Glenn palliation in an infant weighing $7.0 \mathrm{~kg}$, including the result after recanalisation and stenting. $(\mathrm{G} / \mathrm{H})$ Percutaneous pulmonary valve implantation via the right jugular vein in a $16 \mathrm{~kg}$ child.

head and neck vessels are usually larger than the femoral and pelvic vessels.

This series demonstrates the practical benefit of the upside-down positioning in everyday life in terms of feasibility and handling for different treatment modalities. In fact, for our initial upside-down interventions, we stuck to the standard angiographic orientation, mirroring live fluoroscopy both up-to-down and right-to-left. This, however, proved to be very cumbersome because the double mirroring made manoeuvring, steering and probing much more difficult. Without mirroring, a rightturn remains a right-turn and vice versa. Applying the non-mirrored technique practically, we got the impression that we were reaching the target more accurately, even though this breaks with the usual X-ray convention.

There are still other benefits of upside-down positioning: from the technical point of view: there is more workspace behind the $180^{\circ}$-rotated patient's head, simplifying the sterile use of long sheaths, exchange wires and delivery systems. On the other hand, the arrangement of the anaesthesia and monitoring devices, the infusion lines and accessibility to the airways is disadvantageous when carrying out interventions from the neck. Our experience has shown, however, that most of these obstacles can be overcome. The adjacent, side-by-side positioning of the $\mathrm{PO}$ and first assistant when intervening in upside-down position (vs across-corners in conventional positioning) led to a more familiar, straightforward workflow, and it may ultimately enhance patients' safety.

Although operators are exposed to lower levels of radiation than patients, they remain repeatedly exposed, resulting in potentially high lifetime exposure. The harmful effects of radiation exposure in cardiac catheterisation laboratories are well-documented, entailing an increased risk of skin lesions, cataracts and cancer. ${ }^{10-13}$ Over recent years, more sophisticated equipment and the application of radiation safety protocols and programmes have significantly reduced operator exposure ${ }^{14-16}$ and were jointly endorsed by the society for cardiac angiography and interventions. ${ }^{17}$ New radiation technologies and procedural modifications also enhance radiation reduction. ${ }^{1819}$

In this series, operators' radiation exposure was significantly reduced in a bench-test trial in both paediatric and adult phantoms. This approach leads to an ambient dose equivalent reduction of $65.5 \%-97.8 \%$ for the PO.

Table 3 Comparison of the ambient dose equivalent (nSV) for conventional and upside-down positioning in different models averaged over all three angulations

\begin{tabular}{|c|c|c|c|c|c|c|c|c|c|}
\hline Model & Position & PO & $P$ value & Assistant & $P$ value & Nurse & $P$ value & Anaesthetist & $P$ value \\
\hline \multirow[t]{2}{*}{ Adult } & Conventional & $2569 \pm 807$ & $<0.01$ & $61 \pm 54$ & $<0.01$ & $15 \pm 9$ & 0.41 & $8 \pm 6$ & 0.26 \\
\hline & Upside-down & $135 \pm 23$ & & $134 \pm 60$ & & $14 \pm 7$ & & $10 \pm 6$ & \\
\hline Paediatric & Upside-down & $121 \pm 56$ & & $57 \pm 30$ & & $2 \pm 4$ & & $2 \pm 5$ & \\
\hline Spherical & Conventional & $3907 \pm 883$ & $<0.01$ & $100 \pm 70$ & $<0.01$ & $21 \pm 7$ & 0.85 & $12 \pm 5$ & 0.17 \\
\hline
\end{tabular}




\begin{tabular}{lllllllllll}
\hline Table 4 & Comparison of the ambient dose equivalent (nSV) at different projection angles & & & \\
\hline Model & View & Position & PO & P value & Assistant & P value & Nurse & P value & Anaesthetist & P value \\
\hline Adult & PAlateral & Conventional & $1632 \pm 109$ & $<0.01$ & $17 \pm 8$ & $<0.01$ & $6 \pm 6$ & 0.68 & $4 \pm 6$ & 0.68 \\
& & Upside-down & $145 \pm 14$ & & $116 \pm 39$ & & $7 \pm 6$ & & $5 \pm 6$ & \\
& Cranial-LAO/ & Conventional & $3540 \pm 196$ & $<0.01$ & $42 \pm 5$ & $<0.01$ & $24 \pm 4$ & 0.04 & $12 \pm 4$ & 1 \\
& lateral & Upside-down & $106 \pm 6$ & & $175 \pm 83$ & & $19 \pm 6$ & & $12 \pm 4$ & \\
& Cranial-RAO/ & Conventional & $2534 \pm 123$ & $<0.01$ & $123 \pm 51$ & 0.59 & $15 \pm 6$ & 1 & $7 \pm 6$ & 0.19 \\
& lateral & Upside-down & $152 \pm 10$ & & $113 \pm 25$ & & $15 \pm 6$ & & $11 \pm 6$ & \\
Paediatric & PAlateral & Conventional & $224 \pm 11$ & $<0.01$ & $9 \pm 5$ & $<0.01$ & $0 \pm 0$ & 0.35 & $1 \pm 4$ & 1 \\
& & Upside-down & $127 \pm 41$ & & $50 \pm 17$ & & $1 \pm 4$ & & $1 \pm 4$ & \\
& Cranial-LA0/ & Conventional & $468 \pm 20$ & $<0.01$ & $30 \pm 16$ & 0.09 & $2 \pm 5$ & 1 & $2 \pm 5$ & 0.35 \\
& lateral & Upside-down & $179 \pm 11$ & & $52 \pm 25$ & & $2 \pm 5$ & & $1 \pm 4$ & \\
& Cranial-RAO/ & Conventional & $360 \pm 23$ & $<0.01$ & $34 \pm 20$ & 0.02 & $1 \pm 4$ & 1 & $4 \pm 6$ & 0.59 \\
& lateral & Upside-down & $57 \pm 14$ & & $67 \pm 43$ & & $1 \pm 4$ & & $5 \pm 6$ & \\
Spherical & PA/lateral & Conventional & $4184 \pm 234$ & $<0.01$ & $124 \pm 89$ & 0.06 & $19 \pm 6$ & 0.17 & $9 \pm 5$ & 0.17 \\
& & Upside-down & $137 \pm 8$ & & $316 \pm 211$ & & $15 \pm 5$ & & $11 \pm 0$ & \\
& Cranial-LA0/ & Conventional & $4781 \pm 194$ & $<0.01$ & $47 \pm 5$ & $<0.01$ & $26 \pm 6$ & 0.28 & $15 \pm 5$ & 0.55 \\
& lateral & Upside-down & $111 \pm 4$ & & $281 \pm 163$ & & $22 \pm 6$ & & $14 \pm 5$ & \\
& Cranial-RA0/ & Conventional & $2754 \pm 65$ & $<0.01$ & $130 \pm 58$ & 0.38 & $19 \pm 6$ & 0.35 & $11 \pm 0$ & 0.08 \\
& lateral & Upside-down & $129 \pm 10$ & & $165 \pm 86$ & & $22 \pm 6$ & & $15 \pm 6$ & \\
\hline
\end{tabular}

$\mathrm{LAO}$, left anterior oblique; RAO, right anterior oblique.

This is remarkable because not all body parts can be leadprotected, and there is evidence that unprotected parts of the body carry an increased risk of certain cancers. ${ }^{20-23}$ Most cardiac catheter laboratories are set up so that the PO stands on the patient's right side. Most fixed radiation barriers are therefore positioned accordingly. When patients are rotated by $180^{\circ}$ lying on their back, the distance to the X-ray source, especially for the PO, may increase while employing lateral protective barriers (lead glass panes and table-suspended drapes). The greater distance between the X-ray sources and dosimeter leads to a disproportionate decrease in radiation exposure, since the inverse square law applies. ${ }^{24}$

The primary mechanism of radiation exposure during cardiac catheterisation is the scattered radiation caused by the patient himself as a scattering body, with the radiation amount being body surface-dependent. ${ }^{13} 1824$ In this series, the PO's radiation exposure during conventional positioning is about 10 times higher in the adult phantom than in the paediatric phantom. It is noteworthy that this additional burden disappears by switching to upsidedown positioning (in that setting, the PO's radiation exposure was equally low in all phantoms).

We emphasised that the upside-down position leads to a change in the spatial relations between patient, operator and radiation source, entailing angulations that differ from that of conventional set-up. The tube angulation affects the occupational radiation dose amount to varying degrees. ${ }^{1825}$ Knowledge about rational angulating is an important contribution to radiation protection. ${ }^{1326}$ Cranial-LAO projections are the most radiation-intensive projections for both operators and patients in the conventional set-up because the X-ray source is mobilised closer to the operator and involves higher air Kerma values than during other projections. ${ }^{18}$ Accordingly, our bench test results for cranial-LAO concur with the literatures on radiation exposure for the PO. Of note, angulation effects are barely noticeable in the upside-down positioning, since a considerable ambient dose equivalent reduction was achieved at all angulations, especially for cranial-LAO in the adult phantom.

\section{Limitations}

This study is limited by its retrospective nature of the clinical part, with lack of an adequate control population, and a relatively small clinical study cohort with highly diverse cardiovascular disease patterns. In addition, our new method is limited to highly variable modern cardiac catheter systems and by the positioning of team members like the anaesthetist with the equipment who may not be well-suited for performing procedures from the neck.

\section{CONCLUSION}

By positioning the patient lying on their back in $180^{\circ}$ rotation on the catheter table, catheterisation via neck access can be mandatory in various treatment modalities of all ages with congenital heart defects. When used in routine clinical practice, this approach greatly facilitates handling and interventions. Additionally, the local radiation exposure for the $\mathrm{PO}$ was significantly reduced in the paediatric, and most impressively in the adult phantom, 

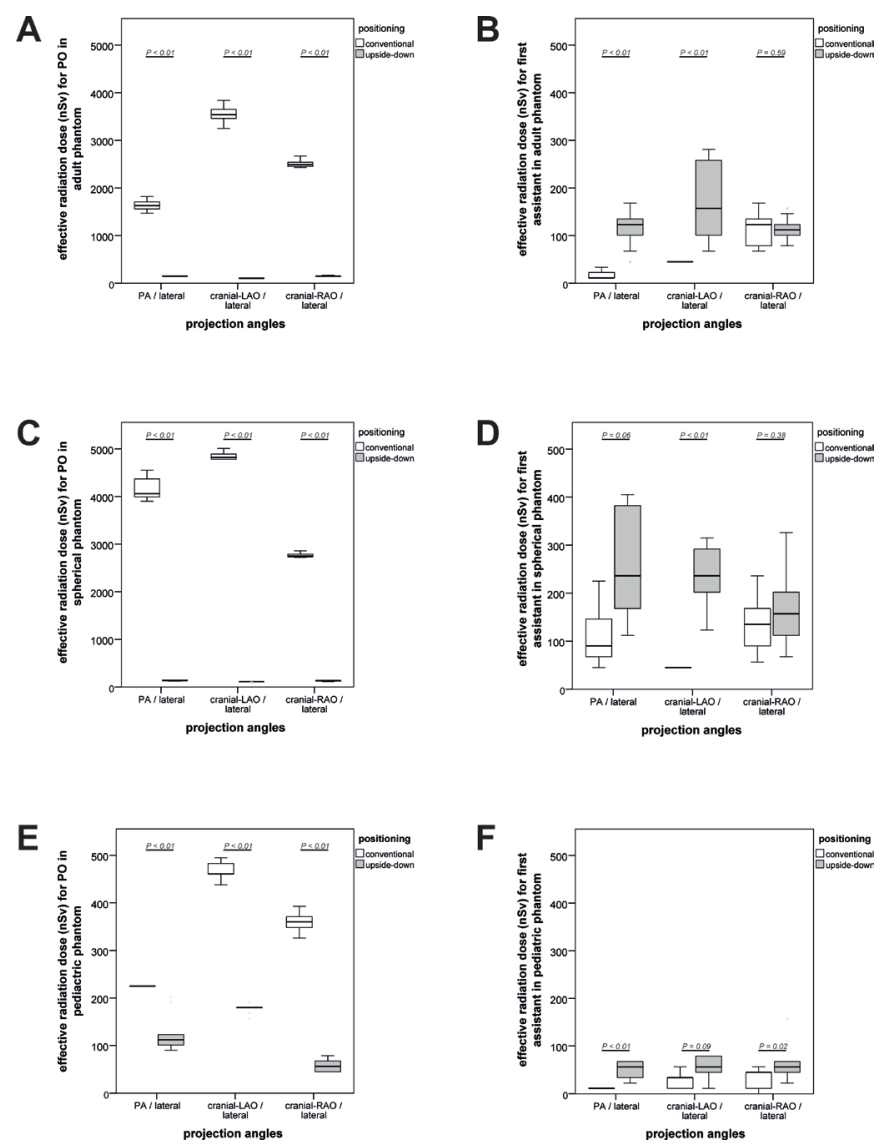

Figure 3 Box-whisker plots illustrating differences in ambient dose equivalents (nSV) for the PO and first assistant in the conventional (white boxes) and the upside-down setup (grey boxes) in adult phantom (A/B), spherical phantom (C/D) and paediatric phantom (E/F). Statistical testing with Mann-Whitney $U$ test. Note the 10-times higher labelling of the $y$-axis section in $(A)$ and $(C)$. $P O$, primary operator.

as demonstrated by bench-test simulation for catheterisation via neck access.

Acknowledgements We thank Claudia Schmoor for statistical advice, Carole Cürten for language editing, John D. Thomson for revising the manuscript, Max Grohmann for photography, Stefan Heinz for graphic design and Elizaveta Stepina from Siemens Healthcare GmbH for providing the Alderson Rando phantom Braden.

Contributors RK and JG developed the concept and design of the study, evaluated the data, interpreted the results and drafted the manuscript. JH and RH were involved in the patient management and they critically revised the manuscript. As radiation protection commissioner, KM took the measurements on the phantoms, evaluated and interpreted the results. BS critically reviewed the manuscript and approved the final manuscript as submitted.

Funding The authors have not declared a specific grant for this research from any funding agency in the public, commercial or not-for-profit sectors.

Competing interests None declared.

Patient consent for publication Not required.

Ethics approval All procedures performed in this study involving human participants were in accordance with the Ethical Standards of the Institutional Committee and with the 1964 Helsinki Declaration and its later amendments or comparable ethical standards. The local Committee for Medical Ethics of the academic centre approved the study and waived the need for informed consent.

Provenance and peer review Not commissioned; externally peer reviewed.

Data availability statement All data relevant to the study are included in the article or uploaded as supplementary information. This study does not report the results of a clinical trial. It is a retrospective data analysis as part of a quality assurance measure.

Open access This is an open access article distributed in accordance with the Creative Commons Attribution Non Commercial (CC BY-NC 4.0) license, which permits others to distribute, remix, adapt, build upon this work non-commercially, and license their derivative works on different terms, provided the original work is properly cited, appropriate credit is given, any changes made indicated, and the use is non-commercial. See: http://creativecommons.org/licenses/by-nc/4.0/.

\section{ORCID iD}

Rouven Kubicki http://orcid.org/0000-0002-9125-8626

\section{REFERENCES}

1 Chessa M, Baumgartner H, Michel-Behnke I, et al. ESC Working group position paper: transcatheter adult congenital heart disease interventions: organization of care - recommendations from a joint Working group of the European society of cardiology (ESC), European association of pediatric and congenital cardiology (AEPC), and the European association of percutaneous cardiac intervention (EAPCI). Eur Heart J 2019;40:1043-8.

2 Kang S-L, Benson L. Recent advances in cardiac catheterization for congenital heart disease. F1000Res 2018;7:370.

3 Davenport JJ, Lam L, Whalen-Glass R, et al. The successful use of alternative routes of vascular access for performing pediatric interventional cardiac catheterization. Catheter Cardiovasc Interv 2008;72:392-8.

4 Martin MH, Shahanavaz S, Peng LF, et al. Percutaneous transcatheter pulmonary valve replacement in children weighing less than 20 kg. Catheter Cardiovasc Interv 2018;91:485-94.

5 Zampi JD, Berman DP, Bocks ML, et al. Factors associated with the internal jugular venous approach for melody ${ }^{\mathrm{TM}}$ transcatheter pulmonary valve implantation. Cardiol Young 2016;26:948-56.

6 Choudhry S, Balzer D, Murphy J, et al. Percutaneous carotid artery access in infants $<3$ months of age. Catheter Cardiovasc Interv 2016;87:757-61.

7 Dahle TG, Kaneko T, McCabe JM. Outcomes following subclavian and axillary artery access for transcatheter aortic valve replacement: society of the thoracic surgeons/American college of cardiology TVT Registry report. JACC Cardiovasc Interv 2019;12:662-9.

8 Dorman SH, Obaid DR. Vascular access and closure for cardiovascular intervention. Heart 2019;105:1279-88.

9 Ligon RA, Ooi YK, Kim DW, et al. Intervention on surgical systemicto-pulmonary artery shunts: carotid versus femoral access. JACC Cardiovasc Interv 2017:10:1738-44.

10 Elmaraezy A, Ebraheem Morra M, Tarek Mohammed A, et al. Risk of cataract among interventional cardiologists and catheterization lab staff: a systematic review and meta-analysis. Catheter Cardiovasc Interv 2017;90:1-9.

11 Harbron RW, Chapple C-L, O'Sullivan JJ, et al. Survival adjusted cancer risks attributable to radiation exposure from cardiac catheterisations in children. Heart 2017;103:341-6.

12 Venneri L, Rossi F, Botto N, et al. Cancer risk from professional exposure in staff working in cardiac catheterization laboratory: insights from the national research council's biological effects of ionizing radiation VII report. Am Heart J 2009;157:118-24.

13 Williams MC, Stewart C, Weir NW, et al. Using radiation safely in cardiology: what imagers need to know. Heart 2019;105:798-806.

14 Badawy MK, Deb P, Chan R, et al. A review of radiation protection solutions for the staff in the cardiac catheterisation laboratory. Heart Lung Circ 2016;25:961-7.

15 Fetterly KA, Bell MR. A practical approach to radiation protection for cardiac catheterization laboratory staff. JACC Cardiovasc Interv 2018;11:213-4

16 Picano E, Vañó E, Rehani MM, et al. The appropriate and justified use of medical radiation in cardiovascular imaging: a position document of the ESC associations of cardiovascular imaging percutaneous cardiovascular interventions and electrophysiology. Eur Heart J 2014;35:665-72.

17 , Hirshfeld JW, Ferrari VA, et al, Writing Committee Members. 2018 ACC/HRS/NASCl/SCAl/SCCT expert consensus document on optimal use of ionizing radiation in cardiovascular imaging-best practices for safety and effectiveness, part 2: radiological equipment operation, dose-sparing methodologies, patient and medical personnel protection. Catheter Cardiovasc Interv 2018;92:2829-55.

18 Agarwal S, Parashar A, Bajaj NS, et al. Relationship of beam angulation and radiation exposure in the cardiac catheterization laboratory. JACC Cardiovasc Interv 2014;7:558-66. 
19 Corrigan FE, Hall MJ, Iturbe JM, 3rd HMJ, et al. Radioprotective strategies for interventional echocardiographers during structural heart interventions. Catheter Cardiovasc Interv 2019;93:356-61.

20 Karatasakis A, Brilakis HS, Danek BA, et al. Radiation-Associated lens changes in the cardiac catheterization laboratory: results from the IC-CATARACT (CATaracts attributed to radiation in the CaTh lab) study. Catheter Cardiovasc Interv 2018:91:647-54.

21 Kitahara CM, Linet MS, Balter S, et al. Occupational radiation exposure and deaths from malignant intracranial neoplasms of the brain and CNS in U.S. radiologic technologists, 1983-2012. AJR Am $J$ Roentgenol 2017;208:1278-84.

22 Ko S, Kang S, Ha M, et al. Health effects from occupational radiation exposure among fluoroscopy-guided interventional medical workers: asystematic review. J Vasc Interv Radiol 2018;29:353-66.
23 Velazquez-Kronen R, Borrego D, Gilbert ES, et al. Cataract risk in US radiologic technologists assisting with fluoroscopically guided interventional procedures: a retrospective cohort study. Occup Environ Med 2019;76:317-25.

24 Durán A, Hian SK, Miller DL, et al. Recommendations for occupational radiation protection in interventional cardiology. Catheter Cardiovasc Interv 2013;82:29-42.

25 Christopoulos G, Makke L, Christakopoulos G, et al. Optimizing radiation safety in the cardiac catheterization laboratory: a practical approach. Catheter Cardiovasc Interv 2016;87:291-301.

26 Chambers CE, Fetterly KA, Holzer R, et al. Radiation safety program for the cardiac catheterization laboratory. Catheter Cardiovasc Interv 2011;77:546-56. 